\title{
Trim and Structural Optimization of Subsonic Transport Wings using Nonconventional Aeroelastic Tailoring
}

\author{
Bret K. Stanford ${ }^{1}$ \\ NASA Langley Research Center, Hampton, VA, 23681 \\ Christine V. Jutte ${ }^{2}$ \\ Craig Technologies, Inc., Cape Canaveral, FL, 32920
}

\begin{abstract}
Several minimum-mass aeroelastic optimization problems are solved to evaluate the effectiveness of a variety of novel tailoring schemes for subsonic transport wings. Aeroelastic strength and panel buckling constraints are imposed across a variety of trimmed maneuver loads. Tailoring with metallic thickness variations, functionally graded materials, composite laminates, tow steering, and distributed trailing edge control effectors are all found to provide reductions in structural wing mass with varying degrees of success. The question as to whether this wing mass reduction will offset the increased manufacturing cost is left unresolved for each case.
\end{abstract}

\section{Introduction}

$\mathrm{T}$ his work is part of NASA's Subsonic Fixed Wing project (within the Fundamental Aeronautics program), which evaluates the efficacy of a broad range of multidisciplinary tools, methods, and technologies towards improving the performance of future subsonic transport aircraft. Targeted design advancements include a reduction in fuel burn, which may be implicitly achieved from a reduction in structural wing weight or a decrease in aerodynamic drag: the former is considered here. An optimal configuration will likely utilize a high-aspect ratio wing (for a reduction in induced drag) operating at minimum mass. As such, a key challenge is to control the aeroelastic behavior of such a highly-flexible system, either passively or actively, by precisely tailoring the load paths through the wing. Formal aerostructural optimization, spread over a collection of realistic load cases and flight metrics, is the best way to exploit the fundamental conflict between wing weight and aeroelastic performance.

Wing structural configurations that have been recently enabled by advances in manufacturing and materials are one focus of this paper. These methods are not typically used in current aircraft structural design processes; the important question is whether they allow for enough of a reduction in wing weight (while satisfying a large set of aeroelastic performance constraints) to offset the increased manufacturing costs. One such method may allow for highly-detailed and localized thickness variations throughout the entire wing skin, via additive manufacturing methods such as electron beam freeform fabrication $\left(\mathrm{EBF}^{3}\right)$ [1]. Functionally graded metals (FGM) may also be considered, where a single structural member is composed of a distribution of two metals, with the material fraction spatially blended from one location to the next [2]. Finally, certain metallic wing components may be replaced with composite members. While laminated wing structures are well within current aircraft design practices [3], automated fiber tow placement machines can now lay laminate fibers along precise curvilinear paths [4]. Tow steering may provide a laminate analogue to the localized metallic thickness variations discussed above. All of these methods are well studied in the literature, though aeroelastic applications are rare. The interested reader is referred to Refs. [5]-[8] for aeroelastic studies with FGM, and Refs. [8]-[11] for details concerning the aeroelastic benefits of composite tow steering.

A second focus of this work is the use of multiple distributed control surfaces along the trailing edge of the transport wing, which can be used to trim the vehicle during a given maneuver load. An example of this is the Variable Camber Continuous Trailing Edge Flap (VCCTEF) system [12]. This technique may seem to be a large conceptual departure from the structural-design methods detailed above, but distributed control surfaces may be equally adept at tailoring the load paths throughout the wing structure. This is done by altering the external airloads on the wing rather than the internal stiffness, but these divisions are blurred in any case due to the coupled aeroelastic nature of the system. The flap deflection scheduling can be optimized such that trim is maintained (constraint) and some objective of interest is minimized: root bending moment [13], deformations at given finite

\footnotetext{
${ }^{1}$ Research Aerospace Engineer, Aeroelasticity Branch, bret.k.stanford@ nasa.gov, AIAA Member.

${ }^{2}$ Research Engineer, Advanced Materials and Processing Branch, christine.v.jutte@ nasa.gov.
} 
element nodes [14], drag, etc. Alternatively, structural design variables may be optimized concurrently with the control surface scheduling [15], either iteratively or simultaneously. This latter scenario is of interest for this work, which will study the interaction between the optimal distribution of material within a flexible wing box, and the optimal scheduling of actuators attached to the trailing edge of that box.

This work is organized as follows. A description of the subsonic transport aircraft and the aeroelastic tools used here are provided first. Then a minimum-mass aeroelastic optimization problem is formulated and solved using simple patch thickness design variables. The various members of the wing box (skins, spars, ribs, stringers) are divided into large design segments [16][17], and the optimal thickness of each segment is found. This type of structural wing design is within the state-of-the-art, and so the resulting design is termed the "baseline", whose mass is repeatedly compared to the minimum mass obtained with more sophisticated aeroelastic tailoring methods. These include methods enabled by additive manufacturing (finely-detailed thickness distributions, and FGM), composite laminates (straight fibers within each design segment, as well as tow steered fibers), and distributed control surfaces.

\section{The Common Research Model}

All of the work in this paper is conducted on the Common Research Model (CRM) seen in Figure 1. This transonic transport configuration has a wing span of $58.7 \mathrm{~m}$, a mean aerodynamic chord of $7.0 \mathrm{~m}$, an aspect ratio of 9 , a taper ratio of 0.275 , a leading edge sweep angle of $35^{\circ}$, and a cruise Mach number of 0.85 . The outer mold line given in Ref. [18] is a $1 \mathrm{~g}$ cruise shape, so an inverse method is used here to obtain the jig shape seen in Figure 1, and used for the entirety of this work. A wing box structure is developed which consists of an upper skin, a lower skin, a leading edge spar (located at 12\% of the local airfoil chord), a trailing edge spar (located at 71\%), 27 ribs, and 6 runout stringers (linearly tapered from a depth of $15 \mathrm{~cm}$ at the root to $5 \mathrm{~cm}$ at the tip). These members are discretized into 92,000 triangular shell finite elements to form a model with 270,000 degrees of freedom.

The structure within the leading and trailing edges of the wing is not explicitly modeled, though an inertial effect is captured with a series of lumped masses attached to the wing box via interpolation elements. These masses, along with similar representations for the engine and the fuel, are also shown in Figure 1. In addition to the inertial loads from the lumped masses, a thrust force is applied to the central engine node. Aerodynamic paneling for the wing, horizontal tail, vertical tail, fuselage, and engine (the latter two represented as cruciforms) is shown in the figure as well, with a total of 4,700 panels. Control surfaces (either along the wing's trailing edge, or the tail's elevator) are not explicitly modeled structurally. Instead, surface deflection is modeled by altering the downwash within the appropriate aerodynamic panels, and the change in aerodynamic loading is implicitly transmitted into the wing structure via the spline-based interpolation methods described below.

\section{Aeroelastic Modeling and Sensitivities}

The shell finite elements used to model the wing structure are defined by a combination of linear strain triangles (LST) and discrete Kirchhoff triangles (DKT) [19]. A vortex lattice method (VLM) [20] is used to model the aerodynamic lifting surfaces, and a finite plate spline (FPS) method [21] is used to transfer downwash and pressures between the aerodynamic and structural modules. Only information pertaining to the wing is transferred in this way: the remaining aerodynamic surfaces are not explicitly tied to any structure. The wing box structure is sized across two different types of maneuvers. The first is a longitudinal maneuver (pull-up, push-over), where the system is trimmed via the angle of attack $\alpha$ and the elevator deflection $\delta$. Following methods outlined in Ref. [22], the trimmed values of these two quantities are automatically found by augmenting the aero-structural coupling equations. The final equation for a longitudinal maneuver is written as:

$$
\left[\begin{array}{cccc}
\boldsymbol{K} & -q \cdot \boldsymbol{Q} & \mathbf{0} & \mathbf{0} \\
-\boldsymbol{P} & \boldsymbol{D}^{s} & -\boldsymbol{L}_{\alpha} & -\boldsymbol{L}_{\delta} \\
\mathbf{0} & q \cdot \boldsymbol{S}_{\boldsymbol{L}}^{T} & 0 & 0 \\
\mathbf{0} & q \cdot \boldsymbol{S}_{\boldsymbol{m}}^{T} & 0 & 0
\end{array}\right] \cdot\left\{\begin{array}{c}
\boldsymbol{u} \\
\boldsymbol{C}_{p} \\
\alpha \\
\delta
\end{array}\right\}=\left\{\begin{array}{c}
N \cdot \boldsymbol{F}^{\text {grav }}+\boldsymbol{F}^{\text {thrust }} \\
\boldsymbol{z}_{x} \\
N \cdot W \\
0
\end{array}\right\}+\left\{\begin{array}{c}
\mathbf{0} \\
\boldsymbol{L}_{\gamma} \cdot \boldsymbol{\gamma} \\
0 \\
0
\end{array}\right\}
$$

The first row of Eq. 1 is the finite element analysis: $\boldsymbol{K}$ is the stiffness matrix, and the solution vector $\boldsymbol{u}$ has six degrees of freedom per node (three displacements and three rotations). Forcing functions include self-weight inertial loading $\boldsymbol{F}^{\text {grav }}$ (scaled by the maneuver load factor $N$, and accounting for both the weight of the wing structure and the lumped masses in Figure 1), thrust loading $\boldsymbol{F}^{\text {thrust }}$ from the engine, and aerodynamic forces. Aerodynamic forces are written as $q \cdot \boldsymbol{Q} \cdot \boldsymbol{C}_{p}$, where $\boldsymbol{C}_{p}$ is a vector of differential pressure coefficients acting on each panel of the vehicle, $\boldsymbol{Q}$ is an interpolation function derived from FPS, and $q$ is the dynamic pressure. 


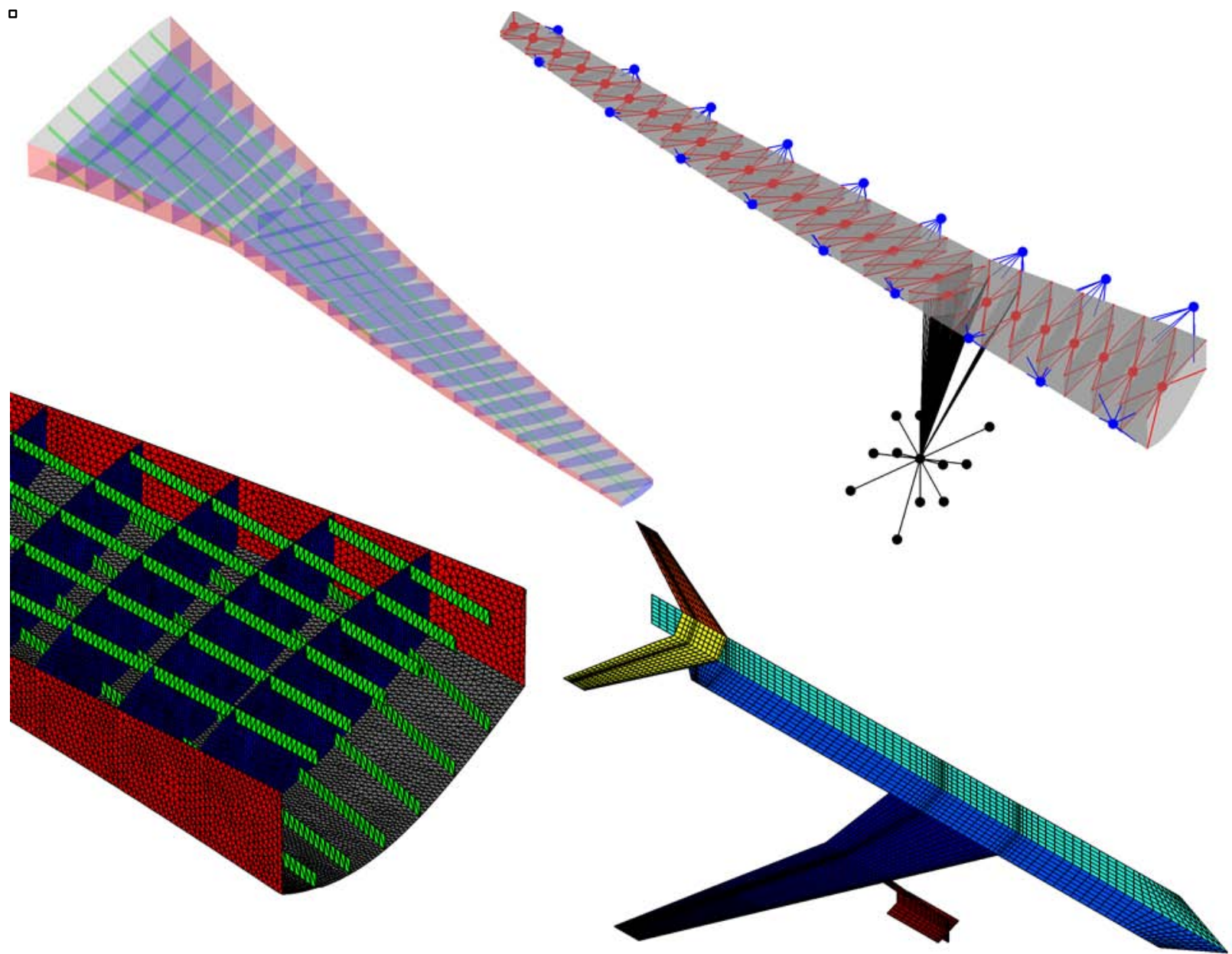

Figure 1. Common Research Model: rib/spar/stringer topology (upper left), lumped-mass representation (upper right), shell-based finite elements (lower left), aerodynamic paneling (lower right).

The second row of Eq. 1 is the aerodynamic analysis, where $\boldsymbol{D}^{S}$ is the aerodynamic influence matrix and the superscript indicates a symmetric aerodynamic condition about the centerline of the airplane in Figure 1 . This equation is driven by several terms: downwash due to angle of attack $\boldsymbol{L}_{\alpha} \cdot \alpha$ (where $\boldsymbol{L}_{\alpha}$ is a linear operator which converts the scalar angle of attack into a downwash at each panel), elevator deflection $\boldsymbol{L}_{\delta} \cdot \delta$, built in camber/twist of the wing and tail jig shapes $\boldsymbol{z}_{x}$, and downwash induced by structural wing deformation. This latter term is written as $\boldsymbol{P} \cdot \boldsymbol{u}$, where $\boldsymbol{P}$ is a second interpolation function, also derived from FPS-based methods. A final downwash term is needed if distributed control surfaces are used along the trailing edge during the maneuver. The deflection of each control surface is grouped into the vector $\boldsymbol{\gamma}$, and $\boldsymbol{L}_{\gamma}$ is a matrix that converts these deflections into the appropriate downwash at each panel.

These deflections $\gamma$ are known, specified quantities during the solution of Eq. 1: $\alpha$ and $\delta$ are found which trim the system in the presence of these and other terms. Trim equations are written in the $3^{\text {rd }}$ and $4^{\text {th }}$ rows of Eq. 1 : $q \cdot \boldsymbol{S}_{L}$ and $q \cdot \boldsymbol{S}_{m}$ convert the differential pressure vector $\boldsymbol{C}_{p}$ into a total aerodynamic lift and aerodynamic pitching moment (about the aircraft center of gravity). Lift must offset the total weight of the vehicle $(N \cdot W)$, and the moment must be zero.

A second type of maneuver considered here is a rolling trim analysis (Eq. 2), where the deflection $\beta$ of an outboard wing aileron is found such that a constant specified non-dimensional roll rate $p \cdot L / U$ is maintained, with no rolling acceleration. In this analysis, $p$ is the dimensional roll rate, $L$ is the semi-span, $U$ is the flight speed, and the aileron is placed between $70 \%$ and $90 \%$ of the semi-span, with a hinge line at $71 \%$ of the local chord. The system is simultaneously trimmed longitudinally for steady level flight $(N=1)$ with the angle of attack $\alpha$. The first analysis requires an anti-symmetric condition about the centerline of the airplane; the second uses a symmetric 
condition. Owing to the linear nature of the methods used here, structural deformations and aerodynamic pressures for the two conditions can be solved separately, and then added together to obtain the total aeroelastic state [22]. The final equation for this maneuver (rolling at level flight) is written as:

$$
\left[\begin{array}{cccccc}
\boldsymbol{K} & \mathbf{0} & -q \cdot \boldsymbol{Q} & \mathbf{0} & \mathbf{0} & \mathbf{0} \\
\mathbf{0} & \boldsymbol{K} & \mathbf{0} & -q \cdot \boldsymbol{Q} & \mathbf{0} & \mathbf{0} \\
-\boldsymbol{P} & \mathbf{0} & \boldsymbol{D}^{s} & \mathbf{0} & -\boldsymbol{L}_{\alpha} & \mathbf{0} \\
\mathbf{0} & -\boldsymbol{P} & \mathbf{0} & \boldsymbol{D}^{a} & \mathbf{0} & -\boldsymbol{L}_{\beta} \\
\mathbf{0} & \mathbf{0} & q \cdot \boldsymbol{S}_{L}^{T} & \mathbf{0} & 0 & 0 \\
\mathbf{0} & \mathbf{0} & \mathbf{0} & q \cdot \boldsymbol{S}_{\boldsymbol{p}}^{T} & 0 & 0
\end{array}\right] \cdot\left\{\begin{array}{c}
\boldsymbol{u}^{s} \\
\boldsymbol{u}^{a} \\
\boldsymbol{C}_{p}^{s} \\
\boldsymbol{C}_{p}^{a} \\
\alpha \\
\beta
\end{array}\right\}=\left\{\begin{array}{c}
\boldsymbol{F}^{\text {grav }}+\boldsymbol{F}^{\text {thrust }} \\
\mathbf{0} \\
\boldsymbol{z}_{x} \\
\left(\frac{p \cdot L}{U}\right) \cdot \boldsymbol{L}_{p} \\
W \\
0
\end{array}\right\}+\left\{\begin{array}{c}
\mathbf{0} \\
\mathbf{0} \\
\boldsymbol{L}_{\gamma} \cdot \boldsymbol{\gamma}^{s} \\
\boldsymbol{L}_{\gamma} \cdot \boldsymbol{\gamma}^{a} \\
0 \\
0
\end{array}\right\}
$$

The $s$ and a superscripts indicate "symmetric" or "anti-symmetric" terms, $q \cdot \boldsymbol{S}_{p}$ converts the aerodynamic pressures into a rolling moment about the centerline (which is ultimately set to zero for a constant roll rate), and $\boldsymbol{L}_{p}$ converts the rolling motion into a downwash term. It is also seen that distributed control surfaces along the trailing edge, if used (in addition to the trimmed aileron deflection $\beta$ ), are also separated into symmetric and anti-symmetric scheduling.

Having solved the trim Eqs. 1 and 2, stresses and strains can be computed for each load case, and a knock-up factor of $30 \%$ is applied to each elemental stress value. A failure function is then computed for each finite element: $\boldsymbol{f}(\boldsymbol{u})$, where $\boldsymbol{u}=\boldsymbol{u}^{s}+\boldsymbol{u}^{a}$ in the case of Eq. 2. For metallic finite elements, the von Mises failure criteria is used, whereas the Tsai-Wu failure criteria is used for laminated composite elements. The wing structure is then divided into patches (seen in Figure 2, with a total of 527 patches), and the Kreisselmeier-Steinhauser function [23] is used to compress all of the elemental failure function values within a given patch into a single metric. If all of the stress values within a patch are within their failure envelope, the $K S$ function for that patch will be less than one.

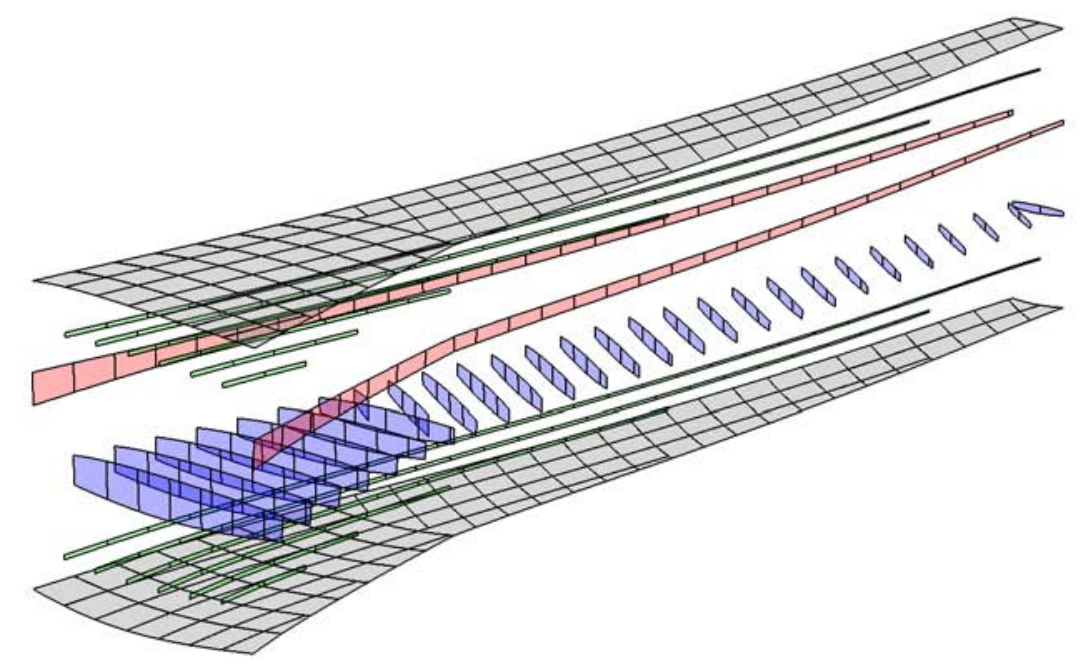

Figure 2. Structural patches used for design variable linking, stress aggregation, and panel identification.

After the stress analysis, a buckling analysis is run for each load case independently. This is done at the panel level, rather than computing buckling factors for the entire wing, in order to reduce the large cost associated with buckling eigenvalue computations, and also as an acknowledgement of the local nature of skin buckling modes. Similar local panel-level analyses (within the context of a wing-level aeroelastic optimization procedure) are also used in Refs. [16] and [24]. These papers use a finite-strip method and a series of analytical shape-function approximations (via Lobatto functions), respectively. The present work employs a full finite element-based buckling analysis within each panel, using sub-structures of the same wing finite element mesh in Figure 1. While more expensive than the previously mentioned alternatives, this approach allows for more accuracy when a spatial distribution of stiffness and/or mass is desired within each panel. 
The structural patches in the upper and lower wing skins of Figure 2 are used as panels (210 total), where each panel is bordered along its perimeter by ribs, spars, or stringers. Each finite element buckling sub-problem is solved with a simply-supported boundary condition along this perimeter:

$$
\left(\boldsymbol{K}_{s}(\boldsymbol{u})+\mu_{n} \cdot \widetilde{\boldsymbol{K}}\right) \cdot \boldsymbol{v}_{n}=\mathbf{0}
$$

where $\boldsymbol{v}_{n}$ is the eigenvector associated with the $n^{\text {th }}$ eigenvalue $\mu_{n}$. The panel stiffness matrix $\widetilde{\boldsymbol{K}}$ is a subset of the wing matrix $\boldsymbol{K}$ used above, but only including the panel finite elements, and constrained by the simply-supported boundary condition around its perimeter. The panel's geometric stiffness matrix $\boldsymbol{K}_{s}$ is assembled with element stresses computed from the global wing deformation vector $\boldsymbol{u}$, as solved in Eqs. 1 or 2. An eigenvalue $\mu_{n}$ greater than one indicates that, for the trimmed aeroelastic state computed in Eqs. 1 or 2, the panel has buckled. Two modes are computed for each panel, and a Kreisselmeier-Steinhauser function [23] is again used to compress the eigenvalues in adjacent panels into a smaller number of constraints.

Several different types of design variables are used in the exercises described below. Thickness-type variables (either for metallic or composite elements) will have an impact on the wing and panel stiffness matrices $\boldsymbol{K}$, $\widetilde{\boldsymbol{K}}$, and $\boldsymbol{K}_{s}$, the inertial loading $\boldsymbol{F}^{g r a v}$, the vehicle weight $W$, and the term $\boldsymbol{S}_{m}$ (which is dependent upon the center of gravity). Certain material-type variables may impact wing/panel stiffness but not mass, and will only have an effect upon the stiffness matrices. In the event that the scheduling of distributed control effectors are used during the optimization, the relevant design variables are $\gamma^{s}$ and $\gamma^{a}$. Derivatives of the aeroelastic response (stress and buckling aggregation parameters for each load case) with respect to these design variables are computed with the adjoint method [25]. This aeroelastic analysis and sensitivity framework is developed as an in-house MATLAB ${ }^{\circledR}$ code. Analysis results have been extensively verified for accuracy against MSC NASTRAN's static aeroelastic solvers, while gradient results are checked against numerical finite differences.

\section{Baseline Model}

Considering an all-aluminum wing $\left(E=70 \mathrm{GPa}, v=0.3, \rho=2780 \mathrm{~kg} / \mathrm{m}^{3}, \sigma_{Y}=330 \mathrm{MPa}\right)$, the optimal distribution of patch-based thickness variables (using the design patches in Figure 2) are found which minimizes the wing mass (based on the volume of the finite element model) subject to aeroelastic strength and buckling constraints:

$$
\begin{aligned}
& { }_{\boldsymbol{x}}^{\min } \text { mass } \\
& \text { s.t. }\left\{\begin{array}{l}
\mathbf{0}<\boldsymbol{x}<\mathbf{1} \\
K S_{f, i} \leq 1 \quad i=1, \ldots,\left(N_{f} \cdot N_{L}\right) \\
K S_{\mu, i} \leq 1 \quad i=1, \ldots,\left(N_{\mu} \cdot N_{L}\right)
\end{array}\right.
\end{aligned}
$$

where $\boldsymbol{x}$ are the thickness design variables which have been appropriately normalized between 0 and 1 : dimensionally, thicknesses are allowed to range between $3 \mathrm{~mm}$ and $20 \mathrm{~mm} . N_{L}$ is the number of load cases, $K S_{f}$ are the stress aggregation parameters $\left(N_{f}\right.$ per load case), and $K S_{\mu}$ are the buckling aggregation parameters $\left(N_{\mu}\right.$ per load case). Three load cases are considered for all optimization problems in this paper. Each has a Mach number of 0.85 and a dynamic pressure of $8.8 \mathrm{kPa}$. The first two load cases are longitudinal maneuvers (Eq. 1), with load factors of 2.5 and -1 , respectively. The third load case is dictated by Eq. 2, where the desired non-dimensional roll rate is 0.08 . All cases are run with half-fuel, and the distributed control surfaces along the trailing edge (except the aileron deflection $\beta$ used for lateral trim) are ignored: $\boldsymbol{\gamma}=\mathbf{0}$.

The design variables $\boldsymbol{x}$ are passed through a linearly-decaying cone-shape filter [26] prior to conversion into structural thickness values, in order to prevent the difference in thicknesses between adjacent patches from being too large. All optimization problems are solved with the Globally-Convergent Method of Moving Asymptotes [27].

The optimal thickness distribution is seen in Figure 3. Only thicknesses in the upper and lower skins are shown, as the optimal rib, spar, and stringer thicknesses are all equal to the lower bound of $3 \mathrm{~mm}$. Upper skins are generally thicker than lower skins, as the pull-up load factor of load case one (2.5g) is greater than the push-over load factor in case two $(-1 \mathrm{~g})$, and the former will induce buckling in the upper skins. Peak thicknesses occur near the trailing edge wing break (where the stresses are highest), and tapers to the lower bound at the tip, where stresses are very low. These stresses are shown in Figure 4, in the form of von Mises-based failure indices. It should be recalled that this 
data is not explicitly exposed to the optimizer, but is first compressed into $K S_{f}$ functions. A failure index greater than 1 indicates excessive aeroelastic stresses, and so these constraints were found to be inactive. The lower skins are generally higher-stressed than in the upper skin due to the thinner members. Skin and spar stresses for the rolling maneuver case are shifted farther outboard (away from the wing break) than the longitudinal cases, as the former has higher torsional loads than the latter. It is also noted that rib stresses are very low, with the exception of the rib attached to the engine, owing to the localized inertial and thrust loading through that attachment.

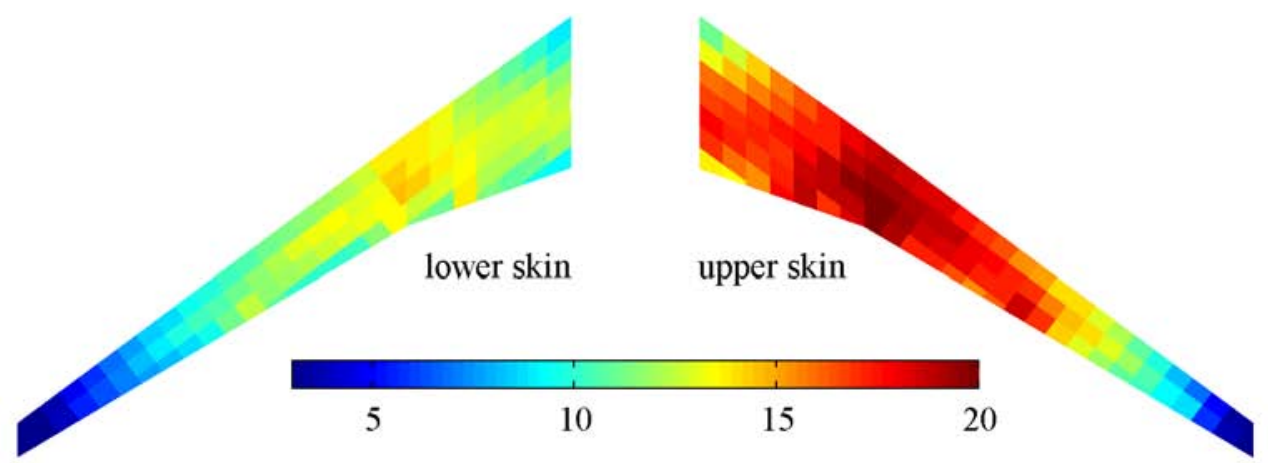

Figure 3. Thickness distribution ( $\mathrm{mm})$ of the baseline design: all rib, spar, and stringer thicknesses are at the lower bound $(3 \mathrm{~mm})$.
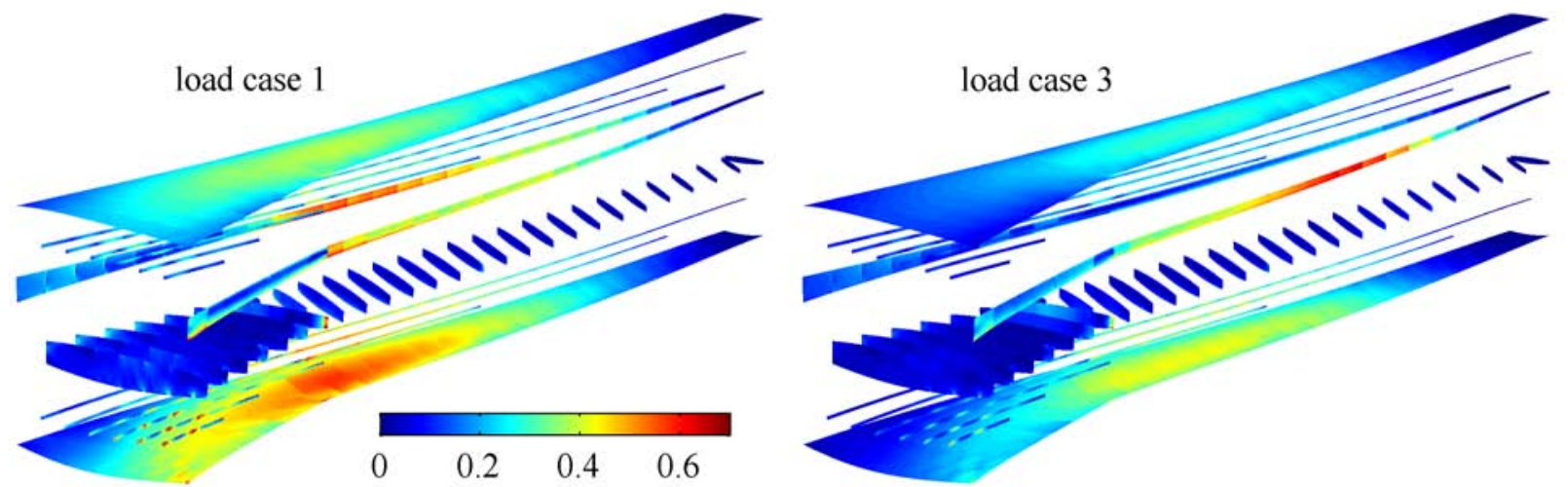

Figure 4. Baseline stress-based failure indices for load case 1 (pull-up maneuver) and 3 (rolling maneuver).

The critical buckling eigenvalues across the panels for each load case are shown in Figure 5. As before, this data is not directly provided to the optimizer, but first compressed into a series of $K S_{\mu}$ functions. Panels with a negative eigenvalue are left blank in this figure, as this is a physically meaningless result: for a given maneuver case, the loads would have to be entirely reversed for these panels to buckle. This clearly happens through most of the lower surface during the positive load factors (cases 1 and 3), and through most of the upper surface during the negative load factor (case 2). Otherwise, many of the panels have a buckling factor nearly equal to one (active constraints), and so the conflict between these $K S_{\mu}$ constraints and the wing mass are driving the design process. For the longitudinal maneuvers, the majority of the wing surface has a large buckling factor, even out towards the wing tip where the stresses (used to form $\boldsymbol{K}_{s}$ in Eq. 3) are very low. Some panels have an active buckling constraint during the rolling maneuver as well, but only in the mid-span location where the stresses in Figure 4 are higher.

The optimal mass of the wing structure (based on the volume of the finite element model, and not including any of the lumped mass in Figure 1) in Figure 3 is $8,521.9 \mathrm{~kg}$. This mass will be repeatedly compared to the minimum mass obtained with more sophisticated aeroelastic tailoring methods detailed below. 


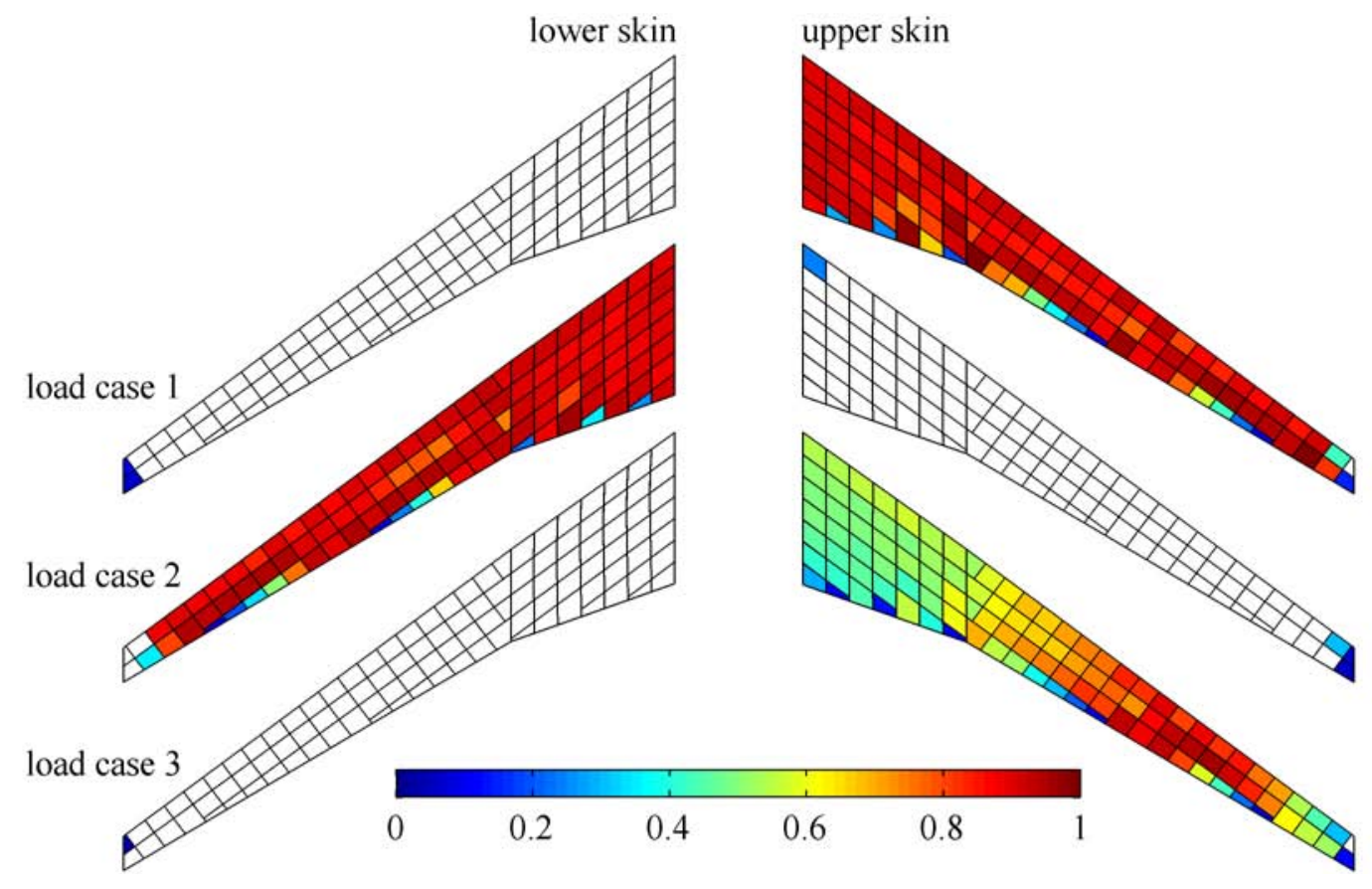

Figure 5. Baseline panel buckling eigenvalues for all three load cases.

\section{Additive Manufacturing}

Two advances upon the previous baseline exercise are considered here. The first allows for highly localized thickness variations throughout the aluminum wing structure, rather than the patch thickness design variables. The second uses FGM, where the optimizer must make simultaneous choices about the thickness and material distribution through the wing structure. Both techniques require the use of additive manufacturing over large acreage structures, which is not commonly used in current aircraft design.

\section{A. Detailed Thickness Variations}

The thickness of every shell finite element is now assigned its own design variable, for 92,000 total design variables, as opposed to 527 for the previous exercise. As noted above, the adjoint method is utilized for the aeroelastic sensitivity analysis, whose computational cost scales weakly with the number of design variables. Cost is proportional to the number of constraints [25], but the $K S_{f}$ and $K S_{\mu}$ details remain unchanged from the previous case. Design variables are again passed through a linearly-decaying cone-shape filter [26] to ensure a smooth thickness distribution. The radius of this filter is $0.3 \mathrm{~m}, 4.2 \%$ of the mean aerodynamic chord.

The optimal thickness distribution for this case is given in Figure 6. Global trends align with that seen for the baseline, with peak thickness at the wing break, minimum thickness at the tip, and thicker panels in the upper skin as compared to the lower. Substantial local variations are evident however, and are clearly driven by panel buckling. Peak thickness lobes are allocated towards the center of each panel, where the buckling eigenvector amplitude is expected to be largest. Panels with two lobes (such as at the leading edge of the root, seen in the inset of Figure 6) would indicate a bi-modal eigenvector as well. Many of the thickness design variables reside at the upper bound of $20 \mathrm{~mm}$; it is expected that allowing these elements to increase their thickness further would allow for a reduction in wing mass, as the optimizer would have more control over the load paths. The mass of this wing is $7888.8 \mathrm{~kg}$, a $7.43 \%$ reduction over the baseline.

\section{B. Functionally Graded Metals}

FGM is computationally realized by assigning material fraction design variables throughout the wing, which vary between $0(100 \%$ material A) and $1(100 \%$ material B). The linear rule of mixtures is then used to compute the elastic modulus, yield stress, and density of the graded metal. Two scenarios are considered for FGM. The first forces the optimizer to make a choice between two metals, one of which is both heavier and stiffer than the other (aluminum and titanium, for example). Potentially, the local increase in mass would stiffen the wing (or alter the 
aeroelastic bend-twist coupling), allowing for a greater reduction in mass elsewhere. For the optimization statement in Eq. 4, however, the optimizer always favored the lighter of the two materials throughout the entire wing. The desire to minimize mass overrides any stiffness benefits, at least for the static aeroelastic physics considered here.

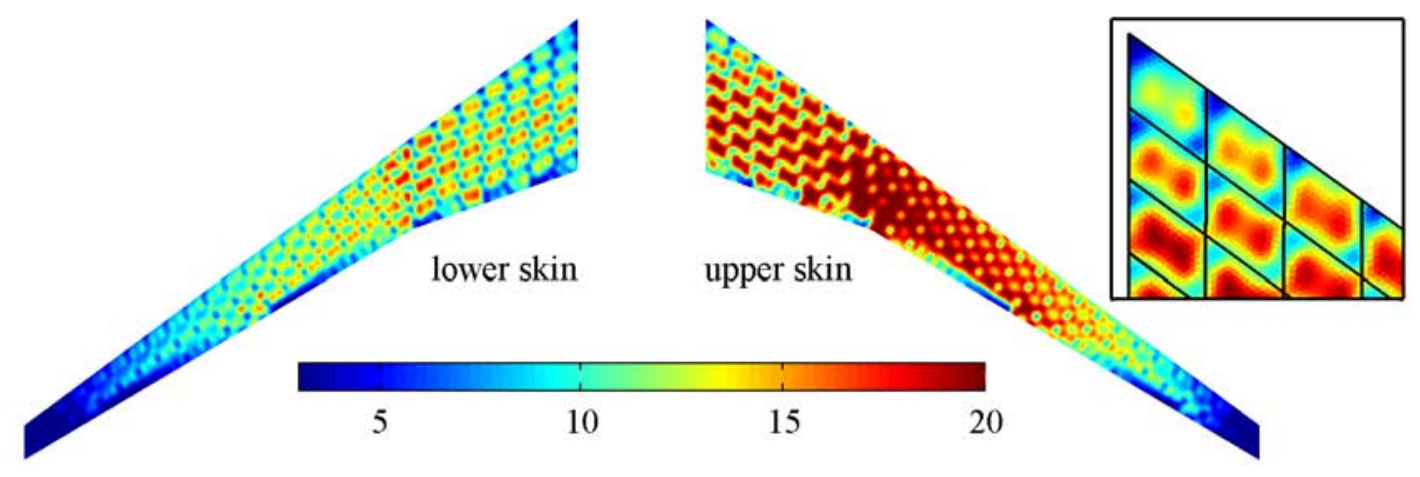

Figure 6. Optimal thickness distribution $(\mathrm{mm})$ when detailed spatial variation are allowed: all rib, spar, and stringer thicknesses are at the lower bound $(3 \mathrm{~mm})$.

The second scenario, which is moderately more successful, uses two metals with identical density, but one is stiffer than the other. This can be accomplished with aluminum (A1), and aluminum with silicon carbide particles (AlSiC), a metal matrix composite. It is to be expected that the optimizer will favor the stiffer AlSiC (since there is no weight penalty) for the majority of the wing, but this design will not provide a fair comparison with the baseline in Figure 3, which is made entirely of aluminum. To facilitate the comparison but still explore the general idea, a fictional material is paired with aluminum, which has the same density, but a 35\% lower modulus and yield stress. The optimal material distribution is given in Figure 7, where a material fraction of 1 is $100 \%$ aluminum, and 0 corresponds to $100 \%$ of the weaker metal. The thickness distribution is also optimized, but looks qualitatively similar to Figure 3, and is not shown.

As expected, the majority of the wing (and almost all of the skins, which bear most of the load) is composed of the stiffer aluminum. But the weaker material is favored in the rear spar, and portions of the ribs. The rear spar design is presumably driven by a desire to increase the negative bend-twist aeroelastic coupling (wash-out), decreasing the overall wing loading [28]. The reasons for the weaker rib material are unclear, as the ribs (except near the engine attachment) bear little load. Regardless, the decrease in mass compared to the baseline is very low: a $0.13 \%$ decrease. It is also noted that the material design variables in Figure 7 are patch-type variables, whereas the true value of FGM comes from a continuous change in material composition. Given the minor improvement seen in this exercise, however, it is not expected that finer variations in material grading will provide substantially better designs.

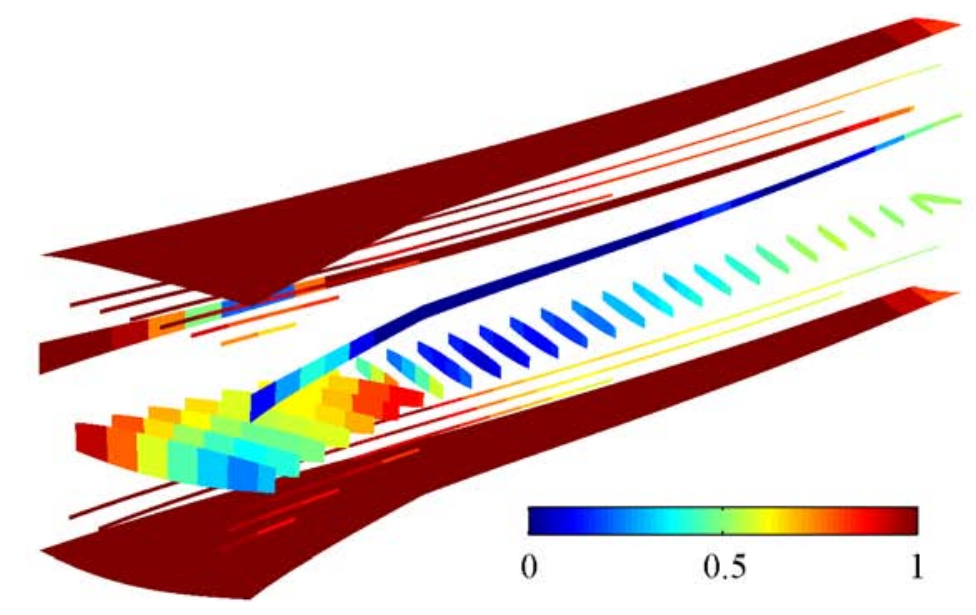

Figure 7. Material fraction distribution: a value of 1 is $100 \%$ aluminum; a value of 0 is $100 \%$ of a weaker material with the same density. 


\section{Composites}

This section uses composite laminates for the upper and lower wing skins; the ribs, spars, and stringers are still composed of aluminum. Composite aircraft structural design is well within the state-of-the-art, of course, but a conventionally-laminated design still must be considered here, in order to provide a fair comparison with tow steered designs. Composite ply material properties are listed in Table 1.

Table 1. Composite ply properties.

\begin{tabular}{|c|c|c|c|}
\hline Parameter & Value & Parameter & Value \\
\hline$E_{1}$ & $128 \mathrm{GPa}$ & $X_{1 t}$ & $1314 \mathrm{MPa}$ \\
\hline$E_{2}$ & $11 \mathrm{GPa}$ & $X_{1 c}$ & $1220 \mathrm{MPa}$ \\
\hline$G_{12}$ & $4.5 \mathrm{GPa}$ & $X_{2 t}$ & $43 \mathrm{MPa}$ \\
\hline$v_{12}$ & 0.28 & $X_{2 c}$ & $168 \mathrm{MPa}$ \\
\hline$\rho$ & $1520 \mathrm{~kg} / \mathrm{m}^{3}$ & $S_{12}$ & $48 \mathrm{MPa}$ \\
\hline$t_{p l y}$ & $0.125 \mathrm{~mm}$ & & \\
\hline
\end{tabular}

\section{A. Conventional Laminates}

Ideally, optimization of composite structures would involve identifying the best laminate stacking sequence for each design patch, though this type of large-scale discrete problem is not amenable to gradient-based optimization. A variety of alternatives are reviewed in Ref. [16], which range from using continuous ply thickness/orientation variables, to the use of lamination parameters. The method proposed by Liu and Haftka [29] is used here, and will be briefly described below. This method recognizes the fundamental separation in roles between the in-plane membrane deformation of a composite skin laminate (dictated by the $\boldsymbol{A}$ constitutive matrix) and the out-of-plane bending deformation ( $\boldsymbol{D}$ matrix). Because the skin thickness is much less than the thickness of the airfoil, global wing deformation properties (deflection, stresses, vibration) are dominated by membrane deformation in the skins. The $\boldsymbol{A}$ matrix is independent of the stacking sequence, only depending on the number of plies at each orientation angle. Panel buckling deformation does depend on the $\boldsymbol{D}$ matrix however (due to the local bending), and so a stacking sequence choice must be made to solve the local problem.

These constitutive matrices can be written as:

$$
\begin{gathered}
\boldsymbol{A}=\left(\boldsymbol{\Gamma}_{0}+V_{1} \cdot \boldsymbol{\Gamma}_{1}+V_{2} \cdot \boldsymbol{\Gamma}_{2}+V_{3} \cdot \boldsymbol{\Gamma}_{3}+V_{4} \cdot \boldsymbol{\Gamma}_{4}\right) \cdot h \\
\boldsymbol{D}=\left(\boldsymbol{\Gamma}_{0}+W_{1} \cdot \boldsymbol{\Gamma}_{1}+W_{2} \cdot \boldsymbol{\Gamma}_{2}+W_{3} \cdot \boldsymbol{\Gamma}_{3}+W_{4} \cdot \boldsymbol{\Gamma}_{4}\right) \cdot h^{3} / 12
\end{gathered}
$$

where $h$ is the thickness of the laminate, $V_{i}$ and $W_{i}$ are lamination parameters, and $\boldsymbol{\Gamma}_{i}$ are material invariants [24]. In this work, ply angles are restricted to $0^{\circ}, 45^{\circ},-45^{\circ}$, and $90^{\circ}$ (where the $0^{\circ}$ direction is aligned with the wing's leading edge), and only balanced, symmetric laminates are considered (specially orthotropic). Because of the balance restriction, a $45^{\circ}$ ply must always be accompanied by a $-45^{\circ}$ ply, and so a logical parameterization is the number of two-ply stacks for each choice: $n_{0}, n_{45}$, and $n_{90}$. The laminate is then a permutation of the form $\left[\left(0^{\circ}\right)_{2 \cdot n_{0}} /\left( \pm 45^{\circ}\right)_{n_{45}} /\left(90^{\circ}\right)_{2 \cdot n_{90}}\right]_{S}$, and the constitutive matrices are written as:

$$
\begin{gathered}
\boldsymbol{A}=\left(\left(n_{0}+n_{45}+n_{90}\right) \cdot \boldsymbol{\Gamma}_{0}+\left(n_{0}-n_{90}\right) \cdot \boldsymbol{\Gamma}_{1}+\left(n_{0}-n_{45}+n_{90}\right) \cdot \boldsymbol{\Gamma}_{3}\right) \cdot 4 \cdot t_{p l y} \\
\boldsymbol{D}=\left(\boldsymbol{\Gamma}_{0}+W_{1} \cdot \boldsymbol{\Gamma}_{1}+W_{3} \cdot \boldsymbol{\Gamma}_{3}\right) \cdot\left(\left(n_{0}+n_{45}+n_{90}\right) \cdot 4 \cdot t_{p l y}\right)^{3} / 12
\end{gathered}
$$

The laminate parameters $V_{2}, V_{4}$, and $W_{4}$ are exactly zero for a specially orthotropic laminate, and $W_{2}$ is small enough to ignore (an approximation which becomes more accurate for thicker laminates). The laminate stiffness is then a function of 5 design variables: $n_{0}, n_{45}, n_{90}, W_{1}$, and $W_{3}$. The two-ply stack variables can be considered as continuous for the purposes of gradient-based optimization (though of course should be rounded to integers for the final design), and variations of the two laminate parameters present a continuous way of changing the stacking sequence, or finding permutations of the laminate $\left[\left(0^{\circ}\right)_{2 \cdot n_{0}} /\left( \pm 45^{\circ}\right)_{n_{45}} /\left(90^{\circ}\right)_{2 \cdot n_{90}}\right]_{S}$ for the purposes of buckling computations. In general, both parameters are bounded [25] by -1 and 1 , and $W_{3}>2 \cdot W_{1}^{2}-1$. For the case where 
the number of $0^{\circ}, \pm 45^{\circ}$, and $90^{\circ}$ stacks are specified, however, $W_{1}$, and $W_{3}$ must also lie within a hexagonal domain [29]. The 6 nodes of this domain are computed from the 6 laminates where the $0^{\circ}, \pm 45^{\circ}$, and $90^{\circ}$ plies are all stacked together.

The minimum-mass optimization process is similar to that used for the baseline, where composite design variables include $n_{0}, n_{45}, n_{90}, W_{1}$, and $W_{3}$ for each of the upper and lower skin panels in Figure 2, and metallic thickness patch design variables in the ribs, spars, and stringers. $K S_{f}$ and $K S_{\mu}$ constraints are spread across the same three load cases, though $K S_{f}$ metrics must now consider Tsai-Wu failure metrics in all four ply orientations for composite panels. Hexagonal constraints must also be included to restrict the choices of $W_{1}$ and $W_{3}$ in each panel, which are written in Ref. [29]. The resulting optimal distribution of two-ply stacks through the skins is seen in Figure 8; all metallic thickness variables in the ribs, spars, and stringers have gone to the lower bound of $3 \mathrm{~mm}$, as above. The mass of this composite wing is $6116.2 \mathrm{~kg}$, a $28.23 \%$ drop from the baseline. Similar mass improvements between metallic and composite wings via aeroelastic optimization have been noted in Ref. [16].

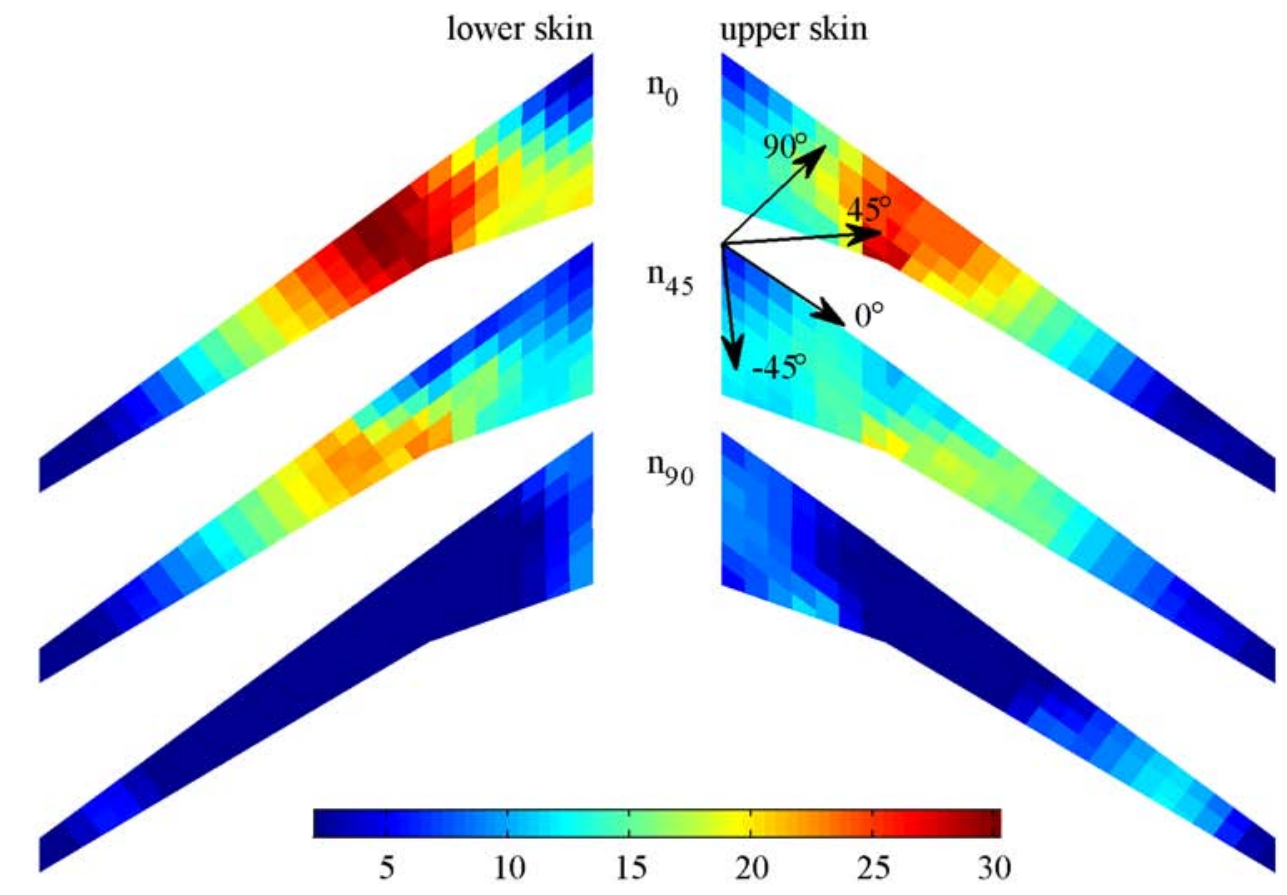

Figure 8. Optimal distribution of 2-ply stacks throughout the wing skins.

The composite thickness distributions mirror the overall trend of the baseline's metallic thicknesses. $0^{\circ}$ are most-prominently used, as expected, with $\pm 45^{\circ}$ shifted further outboard (where torsional stresses are higher, as seen in Figure 4), and some $90^{\circ}$ plies are used at the wing root and tip. Peak thickness of this wing (28 mm) is much higher than used in the metallic baseline $(20 \mathrm{~mm})$, though this is offset by the lower laminate density. $W_{1}$ and $W_{3}$ lamination parameters are shown in Figure 9 for three select panels in the upper skin, along with the hexagonal domains they are forced to lie within. This hexagonal constraint is active for the panel along the trailing edge at the wing break. An inverse process could be conducted to convert each $W_{1}-W_{3}$ pair into an actual laminate stacking sequence, but this is not done here. Contiguous ply constraints (where no more than 4 plies of the same orientation can lie next to each other in the stacking sequence, owing to matrix cracking issues) cannot be included in the laminate parameterization framework used here [29].

\section{B. Tow Steered Laminates}

In this work, separate laminate steering paths are identified for each panel, as shown in Figure 10. A local coordinate system is set up for each panel, whose origin lies at the panel center, with an x-axis parallel to the straight $0^{\circ}$ fibers (parallel to the wing leading edge). Similar to the approach in Ref. [30], the steering angle $\phi$ is defined as a piecewise-linear curve within the panel. Three control points are used here, whose magnitudes are design variables controlled by the optimizer. This results in a curvilinear steering path seen on the right of Figure 10, with constant steering angles along lines perpendicular to the panel's X-axis. 

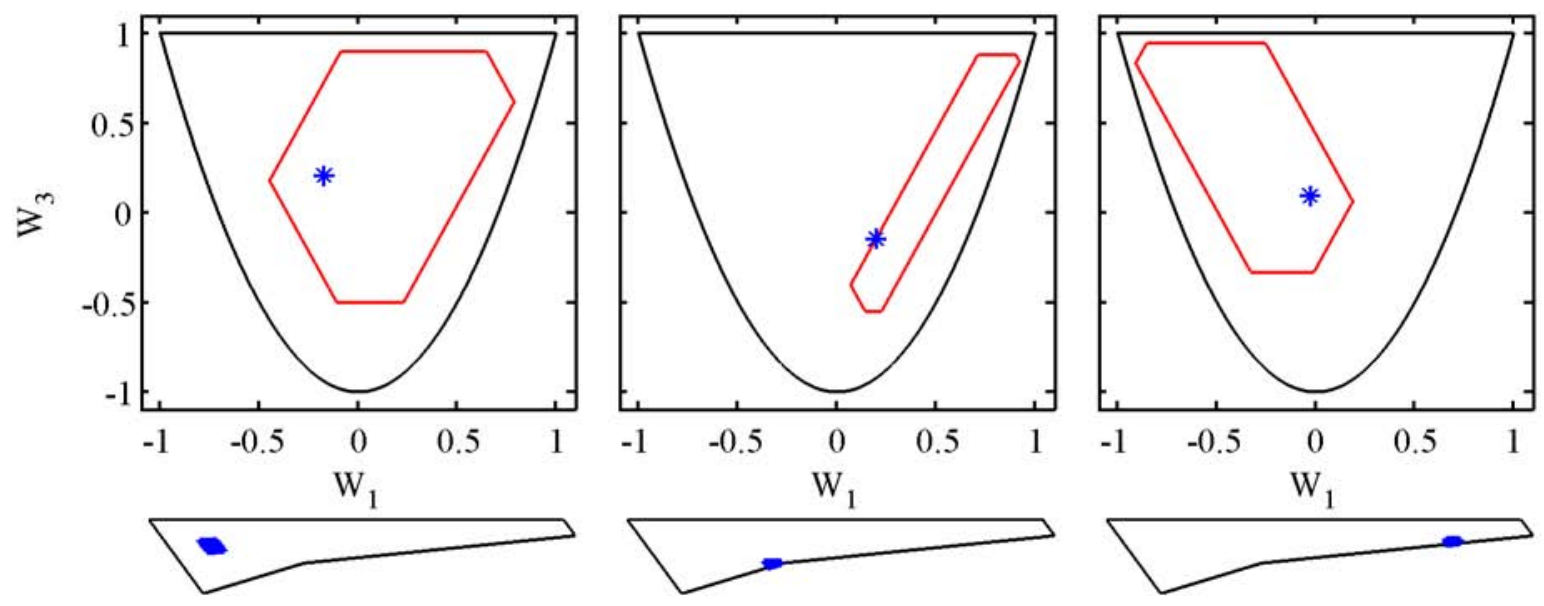

Figure 9. Bending lamination parameters for three panels in the upper skin.

In theory, each ply in a laminate can be steered independently, though the resulting laminate would not, in general, be specially orthotropic. This feature (special orthotropy) is to be preserved in order to continue the use of the parameterization scheme implemented above. A second reason is that, at present, balanced symmetric laminates solely composed of $0^{\circ}, \pm 45^{\circ}$, and $90^{\circ}$ plies are required for certification of commercial transport structures [31]. Specially orthotropic laminates can be utilized in a steering environment by laying all of the $0^{\circ}$ stacks in a laminate along the actual steering path shown in Figure 10. All $90^{\circ}$ stacks are placed along courses perpendicular to this path, and $\pm 45^{\circ}$ layers are similarly steered, seen in Figure 11. Globally, fiber angles can take any value, but locally, each point in each panel has the same laminate (same number of two-ply stacks, same stacking sequence) [31].
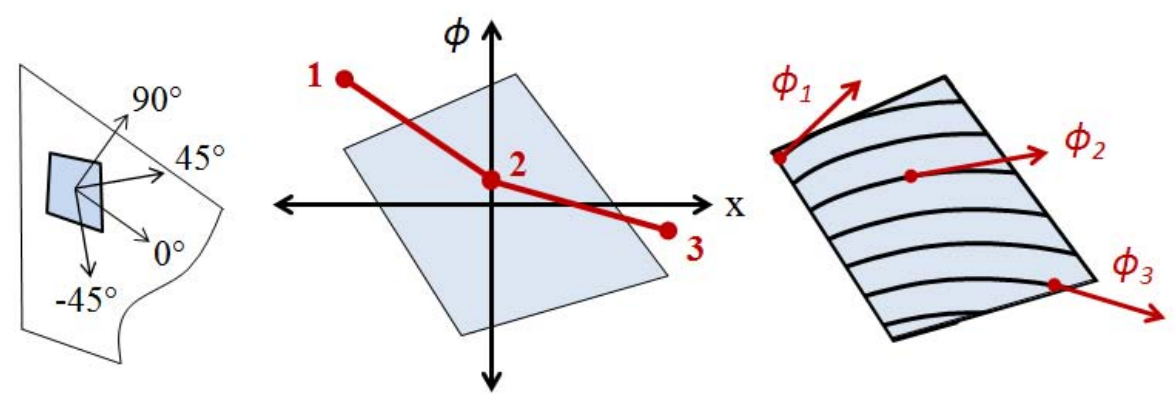

Figure 10. Fiber angles dictated by piecewise-linear splines (three control points per panel), resulting in curvilinear tow paths.

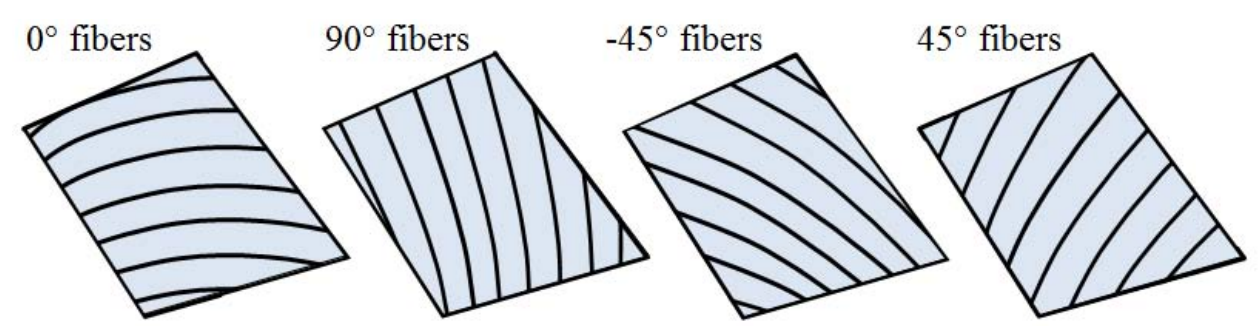

Figure 11. Steering paths of the $0^{\circ}, 45^{\circ},-45^{\circ}$, and $90^{\circ}$ degree fibers, for the example in Figure 10.

The aeroelastic optimization process proceeds in a similar way as in the previous exercise, though the number of design variables per laminated panel is now eight $\left(n_{0}, n_{45}, n_{90}, W_{1}, W_{3}\right.$, and the 3 control points seen in Figure 10), rather than five. The resulting optimal steering patterns in the wing are shown in Figure 12. The remaining design variables are qualitatively similar in overall distribution to the previous result, and will not be shown, but the mass of this tow steered structure is $5,351.7 \mathrm{~kg}$. This is a $37.18 \%$ improvement over the metallic baseline, and 
(perhaps more relevant) a $12.50 \%$ improvement over the conventional laminate result of Figure 8 . Steering path curvature is heavily utilized around the trailing edge wing break of the upper skin, where the parameterization has smoothed the structure around this discontinuity in the wing geometry. This reduces the stress concentration in the region, and allows the optimizer to remove material here. Curvature is also utilized within various panels along the leading edge, and in the outboard portions of the wing.

Some panels, including many at the extreme wing root and tip, do not exhibit curvature; it is unclear whether the dominating physical phenomena in these panels (such as the buckling modes) actually prefer straight fibers, or whether this result is a local-minima. Some of these straight-fibered panels have exploited the tow steering parameterization used here to rotate their entire laminate relative to the leading edge, by using near-equal values for all 3 control points in Figure 10. The fibers are straight, but the $0^{\circ}$ stacks are no longer parallel to the leading edge of the wing. As such, the comparison between this result and the previous $(12.50 \%$ reduction in mass with the advent of tow steering) may not be a fair referendum on the advantages of curvilinear tow paths.

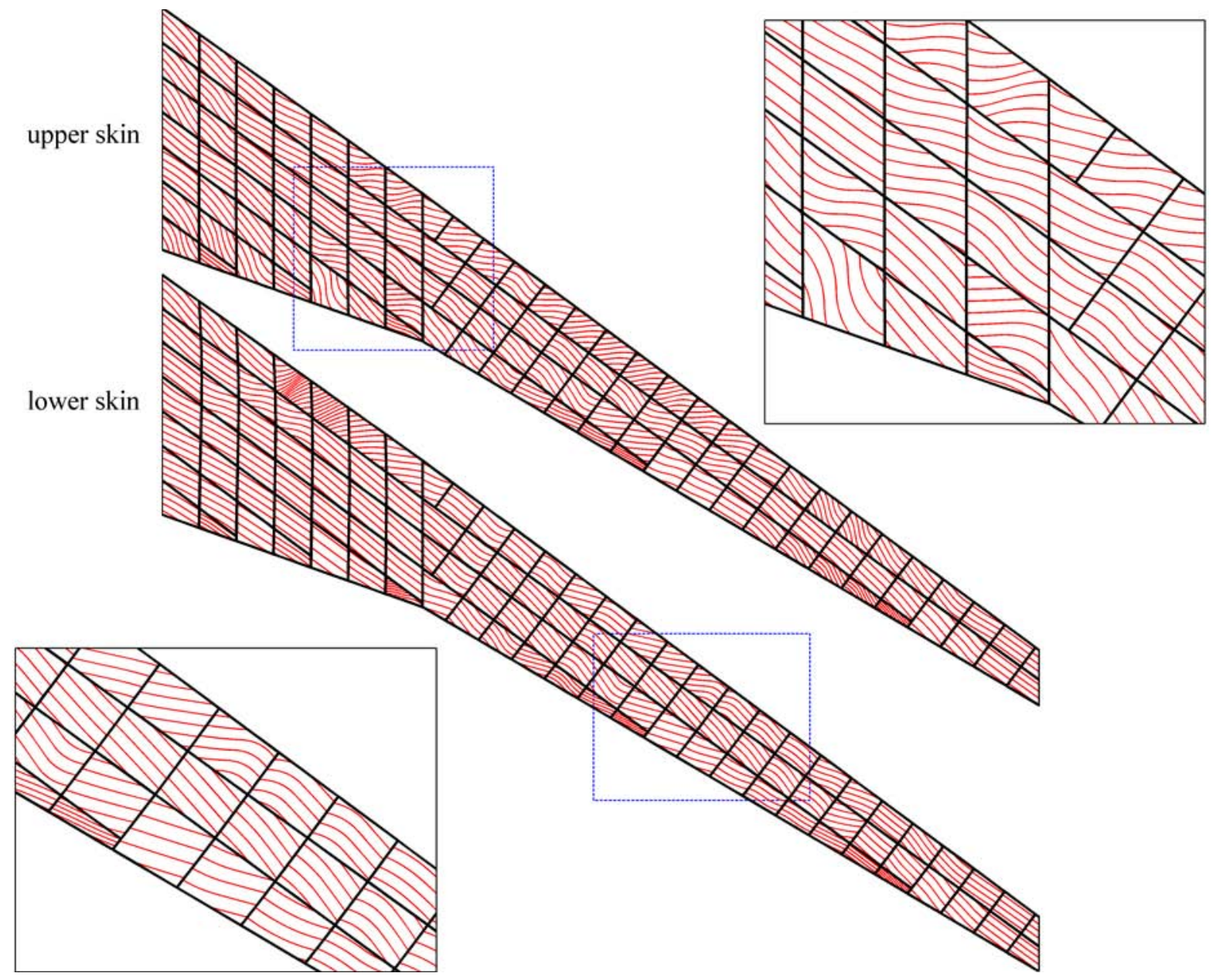

Figure 12. Steering patterns (fiber directions of the $0^{\circ}$ stacks) over the optimal tow steered composite wing.

\section{Distributed Control Surfaces}

The final aeroelastic tailoring scheme returns to the all-metallic patch-based thickness-design-variable methods used for the baseline (Figure 3), but also includes the scheduling of distributed control surfaces along the trailing edge ( $\boldsymbol{\gamma}$ in Eqs. 1 and 2 ) as design variables as well. Simultaneous handling of wing structure design variables and control surface scheduling design variables, within the same optimization loop, is in contrast to most of the existing literature on this topic (see Ref. [15], and papers cited within). Control surface deflections and other trim variables are typically computed within an inner loop (via an over-determined trim optimization process), and structural design variables are optimized through an outer loop. This is due to the limitations of certain codes that can conduct 
structural optimization but not trim optimization. In this work, the use of the adjoint method for aeroelastic gradients enables simultaneous optimization of both sets of design variables, which is presumably more efficient. It should be recalled that, whatever control surface deflections $\gamma$ are imposed by the optimizer, the aeroelastic analysis (Eqs. 1 and 2) will automatically locate the variables ( $\alpha$ and $\delta$ for longitudinal maneuvers, $\alpha$ and $\beta$ for lateral) that trim the aircraft.

The trailing edge of the wing is discretized into 20 control surfaces from root to tip, each with a hinge line at $71 \%$ of the local chord. This results in 80 total control surface design variables: one set each for the longitudinal load cases 1 and 2 (pull-up and push-over maneuvers), and two sets for the rolling load case 3 (a symmetric and an anti-symmetric scheduling). As above, the separation between symmetric and anti-symmetric physics is possible due to the linearity of the system, and total control surface deflections are eventually found by summing $\boldsymbol{\gamma}^{s}$ and $\boldsymbol{\gamma}^{a}$ (or subtracting them, for the left wing). Hinge moment constraints of $\pm 5 \cdot 10^{4} \mathrm{~N} \cdot \mathrm{m}$ are imposed for each control surface, as well as side limits of $\pm 20^{\circ}$. As with structural design variables, the scheduling variables, for a given load case, are passed through a spatial filter in order to maintain a smooth deflection along the trailing edge.

The optimal control surface scheduling is shown in Figure 13 and Figure 14. Positive flap deflection corresponds to a tip-down rotation of the surface, as is conventional [22]. For maneuver load 1 (2.5g pull-up), additional lift is generated in-board, to maintain the overall lift-based trim of the aircraft, but load alleviation is used out-board, in order to reduce the total bending moment on the wing. This allows the optimizer to remove material from the structure (reduce mass, the ultimate objective function) without violating the strength and buckling constraints. Tip-up rotations of all of the control surfaces, from root to tip, would explicitly reduce the stresses and buckling loads even more, but the implicit effect would be an ultimate increase in $\alpha$ to maintain trim, and thus an increase in the aerodynamic loading. The hinge moment constraint is active at the wing root, and the $-20^{\circ}$ side limit is nearly active at the wing break. The spatial filter ultimately prevents the deflection here from truly reaching $-20^{\circ}$, as the filter has the effect of attenuating local rotations.

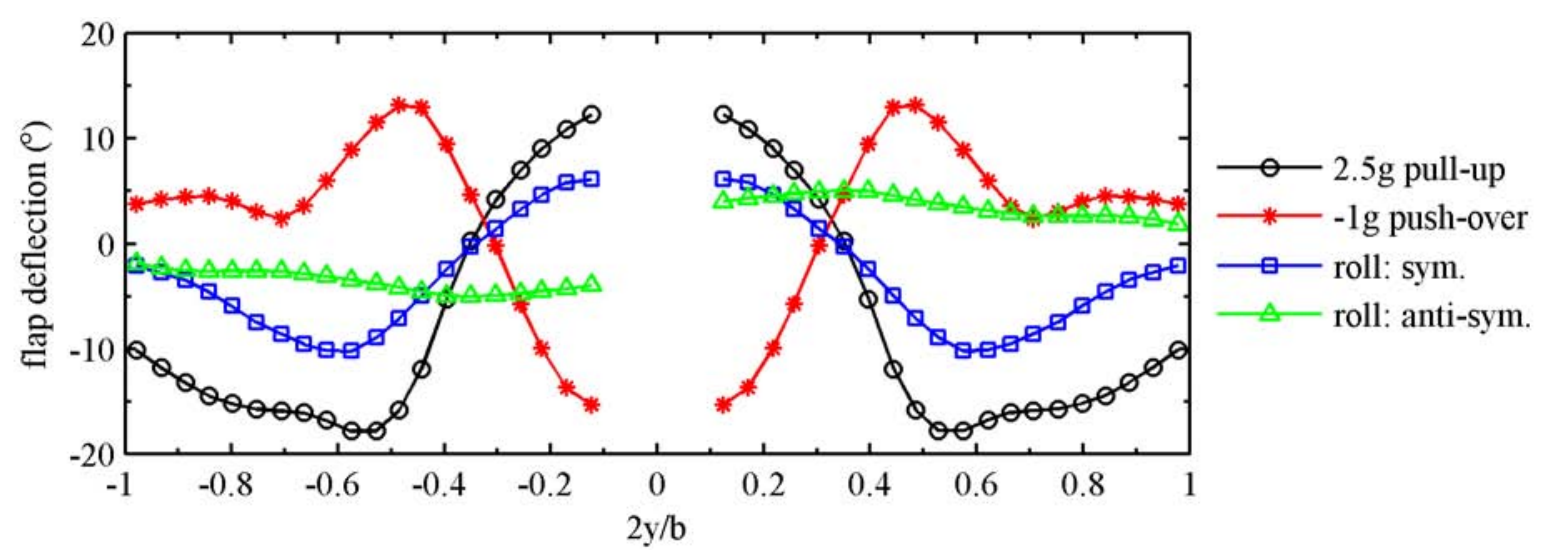

Figure 13. Optimal control surface scheduling for each load case.

Control surface scheduling for load case 2 ( $-1 \mathrm{~g}$ push-over) has the expected opposite trends (but lower magnitude) as compared to the $2.5 \mathrm{~g}$ pull-up maneuver. For load case 3 , the symmetric deflections are responsible (along with the angle of attack $\alpha$ ) for maintaining steady level flight $(N=1)$. As such, the symmetric scheduling has the same trends as load case 1, though the amplitudes are obviously smaller. The anti-symmetric deflections are responsible for maintaining a steady roll rate $(p \cdot L / U=0.08$, as in all the cases in this paper), and so this is accomplished with a moderate tip-down rotation of all the control surfaces, with peak amplitude at the wing break. In-board surface rotations are obviously less effective than outboard at generating a rolling moment, but they impose less torsional stress as well, and so the anti-symmetric scheduling shown in the figures is the optimal compromise.

The optimal patch-based metallic thickness variables are shown in Figure 15: the optimal mass of this structure is $4,571.9 \mathrm{~kg}$, a substantial $46.24 \%$ reduction over the baseline. Overall skin thicknesses have dropped considerably compared to this baseline, with many lower skin panels at the root approaching the lower $3 \mathrm{~mm}$ bound. Peak thickness in the upper skin has been pushed from the wing break (as seen in Figure 3) to the leading edge root. Material is also allocated into the spar structures, the only case in this paper to show such a result. Though not explicitly shown here, the structural deformation of this wing is dominated by torsional deflection (for all three load cases), whereas the other optimal structures display a bending-dominant response. 


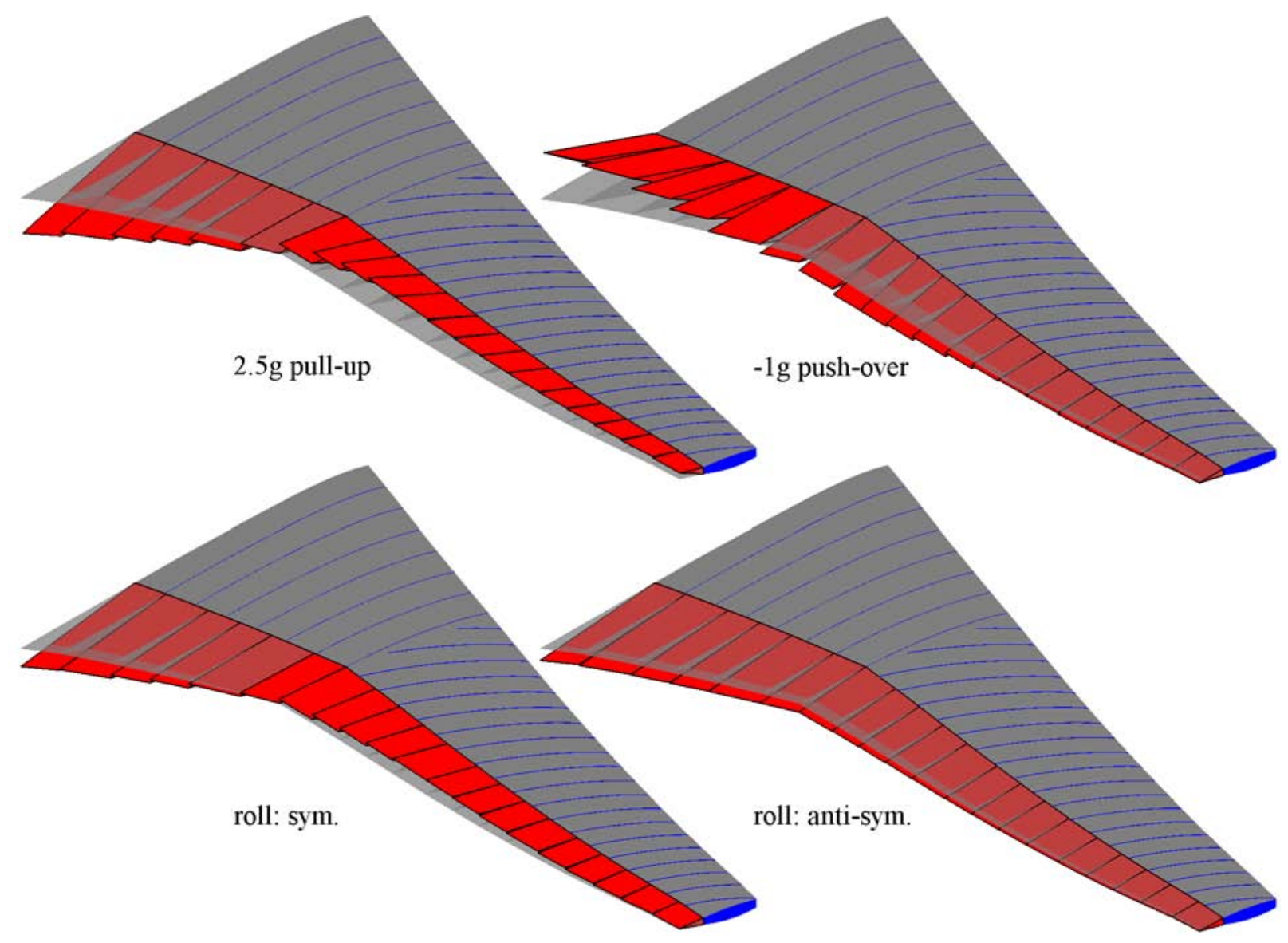

Figure 14. Qualitative picture of control surface schedule corresponding to data in Figure 13.

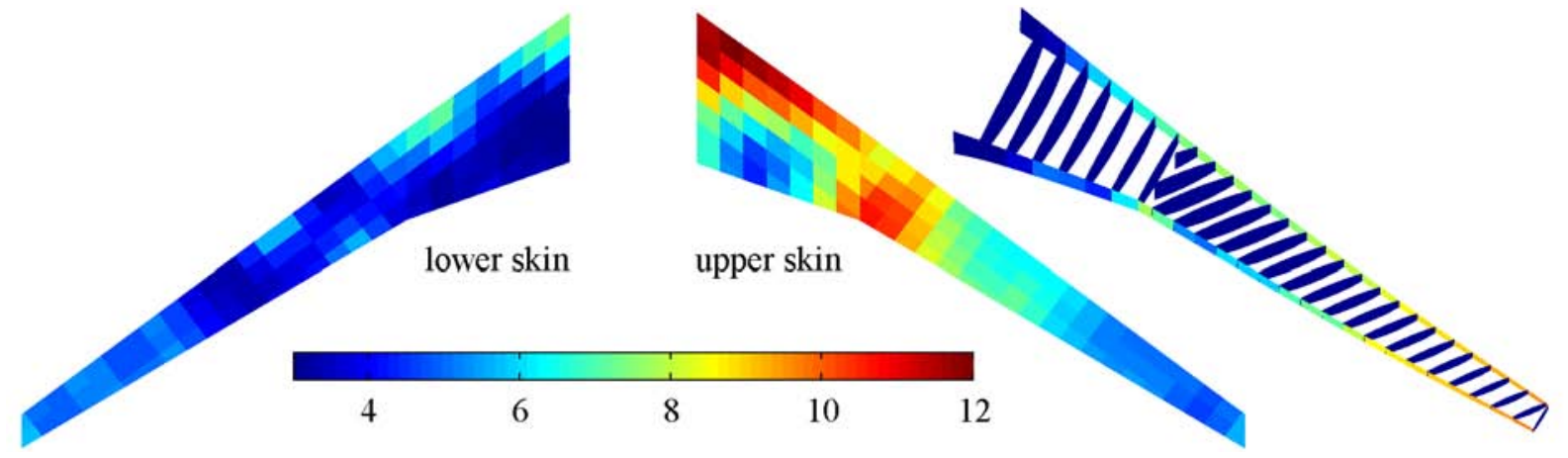

Figure 15. Optimal thickness distribution $(\mathrm{mm})$ for the case where structural thicknesses and control surface scheduling are designed simultaneously.

Perhaps more than any other result in this paper, the optimal structure in Figure 15 should be qualified in terms of modeling omissions, which will surely force an increase in minimum mass. An explicit structural model of each control surface and its mechanism is ignored here, as are the attachment details of each mechanism to the spars. The lumped mass distribution through the trailing edge (seen in Figure 1) is not updated when more control surfaces are included (though each mechanism will be smaller as well, so this perhaps will cancel out). Perhaps most importantly, a flutter constraint is not included in these preliminary results, an aeroelastic phenomena which is known to be exacerbated when material is removed from the root, as is the case in Figure 15. The role of flutter in the aeroelastic optimization process is discussed more in the next section. 
Nonetheless, the exercise in this section has demonstrated the ability to simultaneously optimize both wing structure and control surface scheduling, where the optimizer is able to exploit the conflict between aeroelastic trim and strength/buckling towards a minimum-mass structure.

\section{Conclusions}

An overall summary of the six optimal wing structures presented in this paper is provided in Table 2. All are subject to the same 3 trimmed aeroelastic load cases, under the same set of strength and panel buckling constraints. These minimum-mass optimization studies are entirely enabled by the computation of analytical adjoint-based aeroelastic gradients, which facilitate problems with a large number of structural, material, and flap scheduling design variables (ranging in number from $10^{2}$ to $10^{5}$ ) under a large number of constraints (between $10^{2}$ and $10^{3}$ ).

The lowest wing mass, as compared to the baseline, can be obtained with distributed trailing edge control effectors (46.24\%), though the many caveats associated with this design have been discussed above. A switch in wing skin material from aluminum to composites also enables a sizeable reduction in mass (28.23\%), and the advent of curvilinear tow steering further improves the situation (37.18\%). This is true despite the fact that only balanced specially orthotropic laminates have been considered (for certification reasons, but also to facilitate the chosen parameterization), though unbalanced laminates have long been shown to provide the greatest control over bendtwist coupling [28]. Ref. [24] demonstrates the superiority of unbalanced laminates over balanced for minimum wing mass design. Allowing for a highly detailed metallic thickness distribution through the structure provides a moderate reduction in mass $(7.43 \%)$, but the improvement via FGM is very muted: $0.13 \%$.

Table 2. Summary of optimal wing structures.

\begin{tabular}{|c|c|c|c|}
\hline Description & Structural Wing Mass (kg) & \% Reduction & Figure \\
\hline baseline: patch thickness variables & $8,521.9$ & - & Figure 3 \\
\hline spatially-detailed thickness variations & $7,888.8$ & $7.43 \%$ & Figure 6 \\
\hline functionally-graded metals & $8,508.6$ & $0.13 \%$ & Figure 7 \\
\hline composites: straight laminates & $6,116.2$ & $28.23 \%$ & Figure 8 \\
\hline composites: tow steered laminates & $5,351.7$ & $37.18 \%$ & Figure 12 \\
\hline distributed control surfaces & $4,571.9$ & $46.24 \%$ & Figure 15 \\
\hline
\end{tabular}

A key question with each of these design remains unresolved: is the reduction in wing weight (which will implicitly result in a cost reduction through reduced fuel, among other benefits) enough to offset the increased manufacturing costs? Some of the techniques in Table 2 are far enough beyond the current state-of-the-art (for large acreage structures, in any case) that this question will prove very difficult to answer. Others are perhaps more feasible, and a performance/cost trade-off may be conducted. Manufacturing costs may also be incorporated directly into the optimization process, on a member-by-member basis. For example, on purely physical grounds both panel A and panel B may benefit from the use of an advanced tailoring scheme, but the optimizer may exploit the knowledge that the use of this tool is only warranted in panel B, as the performance improvement in panel A is not enough to offset the manufacturing cost.

This work has taken a relatively small subset of the load cases and design metrics that are typically considered during wing design in industry [3], in an effort to distill the situation down to its fundamental aspects, and make comparative rankings of various aeroelastic tailoring strategies. However, one obvious omission is a dynamic flutter constraint: an explosive and catastrophic failure mode that can play a key role in the design process. Future work will concentrate on the inclusion of flutter constraints. The flutter boundary of each design in Table 2 will be ascertained, and those which overlap with the flight envelope will be re-optimized with this new constraint. Pareto curves will be obtained for those designs which display a particularly strong trade-off between minimum mass and flutter, in an effort to ascertain the potential usefulness of active flutter control-based tailoring schemes.

\section{Acknowledgements}

This work is funded by the Fixed Wing project under NASA's Fundamental Aeronautics Program. 


\section{References}

[1] Taminger, K., Hafley, R., "Electron Beam Freeform Fabrication: A Rapid Metal Deposition Process." Proceedings of the 3rd Annual Automotive Composites Conference, Troy, MI, September 9-10, 2003.

[2] Birman, V., Byrd, L., "Modeling and Analysis of Functionally Graded Materials and Structures," Applied Mechanics Reviews, Vol. 60, No. 5, pp. 195-216, 2007.

[3] Niu, M., Airframe Structural Design, Conmilit Press Ltd., Hong Kong, 1988.

[4] Lukaszewicz, D., Ward, C., Potter, K., "The Engineering Aspects of Automated Prepreg Layup: History, Present and Future", Composites: Part B, Vol. 43, pp. 997-1009, 2012.

[5] Marzocca, P., Fazelzadeh, S., Hosseini, M., "A Review of Nonlinear Aero-Thermo-Elasticity of Functionally Graded Panels," Journal of Thermal Stresses, Vol. 34, pp. 536-568, 2011.

[6] Librescu, L., Maalawi, K., "Material Grading for Improved Aeroelastic Stability in Composite Wings," Journal of Mechanics of Materials and Structures, Vol. 2, No. 7, pp. 1381-1394, 2007.

[7] Dunning, P., Stanford, B., Kim, H., Jutte, C., "Aeroelastic Tailoring of a Plate Wing with Functionally Graded Materials," AIAA SciTech Conference, National Harbor, MD, January 13-17, 2014.

[8] Jutte, C., Stanford, B., Wieseman, C., Moore, J., "Aeroelastic Tailoring of the NASA Common Research Model via Novel Material and Structural Configurations," AIAA SciTech Conference, National Harbor, MD, January 13-17, 2014.

[9] Haddadpour, H., Zamani, Z., "Curvilinear Fiber Optimization Tools for Aeroelastic Design of Composite Wings," Journal of Fluids and Structures, Vol. 33, pp. 180-190, 2012.

[10] Stodieck, O., Cooper, J., Weaver, P., Kealy, P., "Improved Aeroelastic Tailoring Using Tow-Steered Composites," Composite Structures, Vol. 106, pp. 703-715, 2013.

[11] Stodieck, O., Copper, J., Weaver, P., Kealy, P., "Optimisation of Tow-Steered Composite Wing Laminates for Aeroelastic Tailoring," AIAA SciTech Conference, National Harbor, MD, January 13-17, 2014.

[12] Urnes, J., Nguyen, N., Ippolito, C., Totah, J., Trinh, K., Ting, E., "A Mission-Adaptive Variable Camber Flap Control System to Optimization High Lift and Cruise Lift-to-Drag Ratios of Future N+3 Transport Aircraft," AIAA Aerospace Sciences Meeting, Grapevine, TX, January 7-10, 2013.

[13] Raveh, D., "Maneuver Load Analysis of Overdetermined Trim Systems," Journal of Aircraft, Vol. 45, No. 1, pp. 119-129, 2008.

[14] Reich, G., Raveh, D., Zink, P., "Application of Active-Aeroelastic-Wing Technology to a Joined-Wing Sensorcraft," Journal of Aircraft, Vol. 41, No. 3, pp. 594-602.

[15] Zink, P., Mavris, D., Raveh, D., "Maneuver Trim Optimization Techniques for Active Aeroelastic Wings," Journal of Aircraft, Vol. 38, No. 6, pp. 1139-1146, 2001.

[16] Kennedy, G., Martins, J., "A Comparison of Metallic and Composite Aircraft Wings using Aerostructural Design Optimization," AIAA Aviation Technology, Integration, and Operations Conference, Indianapolis, IN, September 17-19, 2012.

[17] Klimmek, T., Development of a Structural Model of the CRM Configuration for Aeroelastic and Loads Analysis," International Forum on Aeroelasticity and Structural Dynamics, Bristol, UK, June 24-26, 2013.

[18] Vassberg, J., DeHaan, M., Rivers, S., Wahls, R., "Development of a Common Research Model for Applied CFD Validation Studies," AIAA Applied Aerodynamics Conference, Honolulu, Hawaii, August 10-13, 2008.

[19] Cook, R., Malkus, D., Plesha, M., Witt, R., Concepts and Applications of Finite Element Analysis, Wiley, New York, 2002.

[20] Katz, J., Plotkin, A., Low-Sped Aerodynamics, Cambridge University Press, Cambridge, UK, 2001.

[21] Appa, K., "Finite-Surface Spline," Journal of Aircraft, Vol. 26, No. 5, pp. 495-496, 1989.

[22] Bisplinghoff, R., Ashley, H., Halfman, R., Aeroelasticity, Addison-Wesley, Cambridge, MA, 1955.

[23] Kreisselmeier, G., Steinhauser, R., "Systematic Control Design by Optimizing a Vector Performance Index", International Federation of Active Controls Symposium on Computer-Aided Design of Control Systems, Zurich, Switzerland, 1979.

[24] Dillinger, J., Klimmek, T., Abdalla, M., Gürdal, Z., "Stiffness Optimization of Composite Wings with Aeroelastic Constraints," Journal of Aircraft, Vol. 50, No. 4, pp. 1159-1168, 2013.

[25] Haftka, R., Gürdal, Z., Elements of Structural Optimization, Kluwer Academic Publishers, Dordecht, The Netherlands, 1992.

[26] Sigmund, O., "Morphology-Based Black and White Filters for Topology Optimization,” Structural and Multidisciplinary Optimization, Vol. 33, pp. 401-424, 2007.

[27] Svanberg, K., "A Class of Globally Convergent Optimization Methods Based on Conservative Convex Separable Approximations," SIAM Journal of Optimization, Vol. 12, pp. 555-573, 2002.

[28] Shirk, M., Hertz, T., Weisshaar, T., “Aeroelastic Tailoring - Theory, Practice, and Promise,” Journal of Aircraft, Vol. 23, No. 1, pp. 6-18, 1986.

[29] Liu, B., Haftka, R., "Single-Level Composite Wing Optimization Based on Flexural Lamination Parameters," Structural and Multidisciplinary Optimization, Vol. 26, pp. 111-120, 2004.

[30] Alhajahmad, A., Abdalla, M., Gürdal, Z., "Optimal Design of Tow-Placed Fuselage Panels for Maximum Strength with Buckling Considerations," Journal of Aircraft, Vol. 47, No. 3, pp. 775-782, 2010.

[31] Wu, K., "Advanced Tow-Steered Composites Technology Development," Proceedings of the NASA Fundamental Aeronautics Program 2012 Technical Conference, Cleveland, OH, March 2012.

[32] Neill, D., Johnson, E., Canfield, R., “ASTROS - A Multidisciplinary Automated Design Tool,” Journal of Aircraft, Vol. 27, No. 12, pp. 1021-1027, 1990. 